\title{
Efecto del deshierbe sobre la transmisión de la escaldadura de la cebada, por ell hongo Rhynchosporium secalis (Oud.) Davis y en la germinación de semillas
}

\author{
Magda Carvajal Moreno, ${ }^{1}$ Enrique Riojas Guadiana ${ }^{2}$ e IGnacio Méndez ${ }^{3}$
}

\begin{abstract}
RESUMEN. Se estudió el efecto del deshierbe en la transmisión de la escaldadura de cebada por semilla y por salpicadura, así como en la germinación de las semillas. Se usaron 10 líneas y 3 variedades de cebada en dos parcelas que tenían cada una 40 lotes de $2 \times 1.5 \mathrm{~m}$, como fuente de semilla y para estudiar el efecto del deshierbe en la transmisión por salpicadura de esporas de una planta enferma a otra sana. Una parcela se dejó con hierbas y la otra se deshierbó. Las semillas de los 80 lotes se colectaron y sembraron en invernadero, para pruebas de germinación y para estudiar la transmisión del hongo por semilla. El área foliar afectada de escaldadura, que se transmitió por agua salpicada, en general fue menos en la parcela enhierbada en el campo, porque el inóculo, o sea las esporas del hongo, tuvo más obstáculos que vencer al saltar de una planta a otra de cebada; pero hubo menos germinación de semillas en este lote. El lote deshierbado tuvo el doble del número de hojas escaldadas y una mejor germinación de semillas en invernadero.
\end{abstract}

ABSTRACT. The effect of weeding on the transmission of barley leaf blotch, as well as on the germination of seeds was studied. Ten genetic lines and three cultivars of barley were used divided in 2 plots, each having 40 blocks of $2 \times 1.5 \mathrm{~m}$ to provide the source of seed, as well as the data about splash transmission of scald. One plot contained weeds and the other did not. The seeds from all the 80 blocks were collected and sown in the glasshouse for ger-

1 Departamento de Botánica, Instituto de Biología, Universidad Nacional Autónoma de México, Apdo. Postal 70-233, Delegación Coyoacán, 04510 México, D. F.

${ }^{2}$ Campo Agrícola Experimental del Valle de México (El Horno) INIA, SARH, Chapingo, Estado de México.

${ }^{3}$ Instituto de Investigación en Matemáticas Aplicadas y en Sistemas, Universidad Nacional Autónoma de México, Delegación Coyoacán, 04510 México, D. F.

Carvajal-Moreno M, Riojas-Guadiana E, Méndez I. 1991. Efecto del deshierbe sobre la transmisión de la escaldadura de la cebada, por el hongo Rhynchosporium secalis (Oud.) Davis y en la germinación de semillas. Boletín de la Sociedad Botánica de México 51: 13-24. 
mination tests and for the study of seed transmission of the fungus. The foliar area affected by leaf blotch and transmitted by splash water, in general was less in the weedy plot in the field, because the inoculum had more obstacles to overcome, jumping from one sick barley plant to a healthy one; thus there was less germination of the seeds from this plot. The clean plot had twice the number of leaf blotch infected plants, and a better rate of seed germination in the glasshouse.

Las enfermedades de los cereales son causadas por gran variedad de microorganismos. Hay que conocer la biología tanto de la planta como del patógeno, así como la relación patogénica, el comportamiento del patógeno en diferentes etapas de la enfermedad y las condiciones de temperatura para realizar una protección efectiva del cultivo.

Debe conocerse la biología de la enfermedad, antes de aplicar cualquier medida de control que pudiera prevenir o suprimir la infección más que curar a las plantas enfermas.

La escaldadura de la cebada es una enfermedad causada por Rhynchosporium secalis, un hongo que se dispersa por salpicadura y por rastrojo infectado (Ozoe, 1956 y Skoropad, 1960) que tiene la habilidad de sobrevivir un año difícil en el rastrojo infectado, o bien en plantas voluntarias, por tanto, actuando como una fuente de infección cuando se siembra un cultivo susceptible.

El término "escaldadura" describe la enfermedad. Los síntomas consisten inicialmente en lesiones ovales azul grisáceas, romboides, de 0.5 a $3 \mathrm{~cm}$ de longitud, que después se tornan café-púrpura con un margen más claro; estas lesiones se pueden observar en las hojas, tallos, vainas y semillas (Habgood, 1971; Kay, 1971; Reed, 1957; Skoropad, 1960 y Smith, 1937).

Las pérdidas de la cosecha son altas, por ejemplo en el suroeste de Inglaterra, las plantas estuvieron afectadas por este hongo, y hubo reportes del $40 \%$ de pérdidas (Ministry of Agriculture, Fisheries and Food, 1972), aunque en brotes más dañinos llegó a un rango de 10 a $25 \%$.

En México, las pérdidas causadas por esta enfermedad son del orden de 15 a $30 \%$, aumentando en tiempo de lluvia (Carvajal, et al., 1986).

El estudio tiene como finalidad el determinar el efecto de las hierbas en la transmisión de la escaldadura de la cebada tanto por semilla, como por salpicadura, y la germinación subsecuente de la semilla infectada.

La transmisión de la escaldadura por semilla es una fuente de inóculo primario frecuente. La presencia de hierbas afecta la transmisión del hongo por agua salpicada (Skoropad, 1959).

Es importante, por tanto, conocer el efecto de las hierbas en la viabilidad de las semillas de la cebada. 


\section{MATERIALES Y MÉTODOS}

Efecto del deshierbe. Para conocer las condiciones iniciales de la semilla con la que se iba a desarrollar el experimento del efecto del deshierbe, se usaron 7 líneas y 3 variedades de cebada. Las líneas fueron: 9554-I, 9589-G, 9591, 9592-G, 9620-B, 9626-B y 9514 A; las variedades de cebada usadas fueron Chevalier, Tlaxcala y Porvenir que son las que más se usan en el Valle de México.

Con objeto de determinar los porcentajes de germinación y transmisión de la escaldadura, se sembraron 100 semillas de cada cebada en charolas en el invernadero; además se pesaron 1000 semillas de cada línea o variedad.

Ya en el campo, se utilizaron dos lotes con un total de 80 parcelas, subdivididos cada uno en 40 parcelas de $2 \times 1.5 \mathrm{~m}$, que fueron sembrados con las 10 líneas/variedades de cebada y sus 4 repeticiones por cada tratamiento. Cada parcela contenía 4 surcos de 2 m de largo y el tipo de siembra fue a chorrillo; un lote con sus 40 parcelas, se deshierbó y el otro con sus 40 parcelas se dejó enhierbado. Se identificaron las especies de hierbas presentes en el lote con las 40 parcelas enhierbadas. Este experimento se realizó en el Campo Agrícola Experimental del Valle de México, "El Horno" del Instituto Nacional de Investigaciones Agrícolas (INIA).

De cada parcela se sacó al azar una muestra de 10 plantas con la cual se determinó el porcentaje de área foliar escaldada y el de semilla infectada. Para esto último se registraron 5 hojas de cada una de las 800 plantas de muestra. Finalmente, se sembraron 10 semillas de cada una de las 80 parcelas, en macetas en el invernadero, para conocer su germinación y el porcentaje de área foliar escaldada en las plántulas después del tratamiento con y sin hierbas.

Análisis estadístico. En la evaluación del experimento de deshierbe, se utilizó un análisis de varianza en el que a los datos de germinación se les aplicó la transformación angular de Bliss (Zar, 1974).

Para encontrar cuáles variedades son estadísticamente diferentes, se realizó la prueba de Tukey para comparaciones múltiples. La "mínima diferencia significativa honesta" (HLSD) es:

$$
\text { HLSD }=Q \sqrt{\text { CM error } / 8}
$$

donde $Q$ es el valor tabular para una $P=0.05$ y 60 grados de libertad para 10 medias de un intervalo de Student; y CM error es el valor de los cuadrados medios atribuible al error, por tanto:

$$
\text { HLSD }=4.646 \frac{\sqrt{\frac{0.0637}{8}}}{8}=0.4145
$$


de modo que si un par de medias de variedades difieren en por lo menos 0.4145 , son estadísticamente distintas (Zar, 1974).

\section{RESULTADOS}

Efecto del deshierbe en la transmisión de la escaldadura. Los resultados de la diseminación de la escaldadura de la cebada en tratamientos con y sin hierbas se presentan en la tabla 1. La variedad Tlaxcala y las líneas 9591, 9592-G y 9626-B mostraron plántulas escaldadas, lo que indica que la semilla "madre" ya traía Rhynchosporium secalis desde antes de sembrarla en el campo; el resto de líneas y variedades estuvieron limpias de escaldadura.

En el campo, en el $70 \%$ de los tratamientos, se registró una mayor cantidad de área foliar escaldada en los lotes limpios, en comparación con los lotes enhierbados (fig. 1); sin embargo, sólo las líneas $9592-\mathrm{G}$ y la $9626-\mathrm{B}$ presentaron una diferencia significativa al $5 \%$ de probabilidad entre sus lotes limpios y enhierbados, como se aprecia en la tabla 1 , donde se presentan en forma conjunta los resultados de los tres experimentos y se muestran los pesos de 1000 semillas de cada cebada. Las líneas 9591 y 9554-I, junto con la variedad Chevalier en tercer lugar, fueron las que más peso tuvieron y esto favorece la producción.

Entre las hierbas o malezas más comúnmente encontradas en los cultivos de cebada en México estuvieron:

1. Quelite (Amarunthus hybridus)

2. Mostaza amarilla (Brassica campestris)

3. Correhuela negra (Convolvulus arvensis)

4. Carretilla (Medicago hispida var. denticulata)

5. Acahual (Encelia mexicana)

6. Media (Madia glomerata)

7. Rábano silvestre (Raphanus raphanistrum)

8. Campanilla o gloria de la mañana (Ipomoea sp.)

9. Avena silvestre (Avena fatua)

En la transmisión de Rhynchosporium secalis a través de las 10 semillas procedentes del campo de cada una de las 80 parcelas, de un total de 400 semillas, 49 semillas de las parcelas limpias transmitieron a $R$. secalis, y sólo 23 semillas de las parcelas enhierbadas lo hicieron (tabla 2). Sobresalen los datos de la línea 9626-B que transmitió muy bien a $R$. secalis a través de la semilla, siguiéndole en efectividad las variedades Tlaxcala, Chevalier, línea 9554-I y Porvenir.

Las condiciones climáticas del experimento se muestran en la (fig. 2), donde se ve que entre el 20 y 30 de enero de 1980 se presentaron condiciones ideales para el ataque 
TABLA 1. Diseminación de la escaldadura de la cebada en tratamientos con y sin hierbas.

\begin{tabular}{|c|c|c|}
\hline ínea o & Diseminación de la escaladura a & $\begin{array}{l}\text { Area foliar escaldada en } \\
\text { campo ( } \ddot{x} \text { de } 400 \text { hojas }\end{array}$ \\
\hline & $\begin{array}{l}\text { dero, antes del tratamiento con y } \\
\text { sin hierbas de la semilla "madre". }\end{array}$ & $\begin{array}{l}\text { de cada línea o variedad } \\
\text { de cebada). }\end{array}$ \\
\hline
\end{tabular}

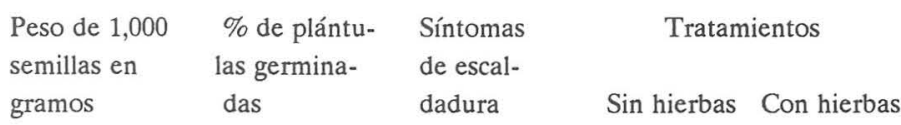

$\begin{array}{lll}\text { 9554-I } & 46.08 & 72 \\ 9589-\mathrm{G} & 37.17 & 71 \\ 9591 & 46.12 & 74 \\ 9592-\mathrm{G} & 31.23 & 78 \\ 9620-\mathrm{B} & 35.83 & 72 \\ 9626-\mathrm{B} & 33.50 & 77 \\ \text { 9514-A } & 44.05 & 72 \\ \text { Chevalier } & 42.23 & 55 \\ \text { Tlaxcala } & 22.87 & 89 \\ \text { Porvenir } & 38.64 & 89\end{array}$

$\begin{array}{cc}- & 9.2 \\ - & 6.2 \\ + & 1.3 \\ + & 11.1^{*} \\ - & 6.3 \\ + & 7.8^{*} \\ - & 13.6 \\ - & 4.8 \\ + & 6.2 \\ - & 8.3\end{array}$

6.4
5.3
1.8
$4.7^{*}$
4.3
$4.3^{*}$
9.6
5.9
6.3
8.2

$\%$ de Area foliar escaldada proveniente de plántulas de 80 semillas de cada variedad en invernadero depués del tratamiento.
Sin hierbas Con hierbas Total del\% de área foliar escalada

* Presentaron diferencia significativa $(\mathrm{P}<0.05)$. 
TABLA 2. Transmisión de Rhynchosporium secalis en invernadero, por semillas provenientes de las parcelas limpias y enhierbadas.

$\begin{array}{lllll}\text { Línea o } & \text { Número de } & \text { Número de } & \text { Número de } & \text { Número de } \\ \text { variedad } & \text { la parcela } & \text { semillas que } & \text { la parcela } & \text { semillas que } \\ & \text { limpia } & \text { transmitieron } & \text { enhierbada } & \text { transmitieron } \\ & & \text { a } \text { R. secalis } & & \text { a } \text { R. secalis }\end{array}$

$\begin{array}{lcccc}9554-\mathrm{I} & 1 & 2 & 71 & 1 \\ 9589-\mathrm{G} & 2,12,22,32 & 0 & 42,52,62,72 & 0 \\ 9591 & 13 & 1 & 43,53,63,73 & 0 \\ 9592-\mathrm{G} & 4,14,24,34 & 0 & 44,54,64,74 & 0 \\ 9520-\mathrm{B} & 25 & 2 & 65 & 1 \\ 9626-\mathrm{B} & 6 & 8 & 46 & 5 \\ & 16 & 4 & 56 & 2 \\ & 26 & 8 & 66 & 3 \\ \text { Porvenir } & 36 & 4 & 76 & 0 \\ \text { Chevalier } & 7 & 2 & 47,57,67,77 & 0 \\ \text { Tlaxcala } & 8 & 2 & 78 & 1 \\ & 29 & 2 & 69 & 5 \\ \text { 9514-A } & 9,19,39 & 0 & 79 & 5 \\ & 20 & 3 & 50,60 & 0 \\ & 30 & 3 & 70 & 0 \\ & 40 & 8 & 80 & 0\end{array}$

Total 


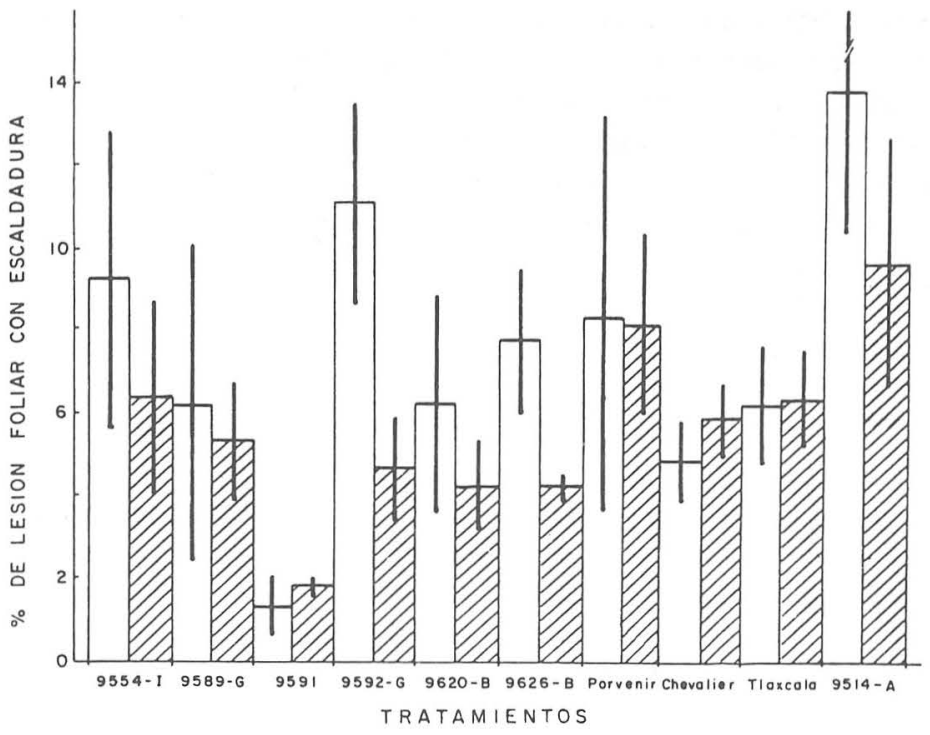

Fig. 1. Media del área foliar afectada (160 hojas) del lote limpio las variedades de cebada en el campo. y el que tenía hierbas

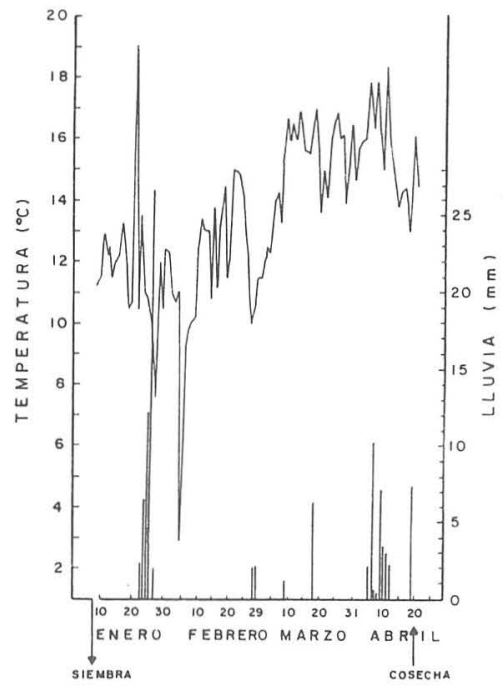

Fig. 2. Condiciones climáticas durante el experimento del efecto del deshierbe sobre la transmisión de la escaldadura de la cebada en 1980. 
de $R$. secalis pues coincide con una alta precipitación pluvial (hasta $26 \mathrm{~mm}$ ) y temperaturas bajas hasta de $7.5^{\circ} \mathrm{C}$; se pudo comprobar este hecho al registrar las hojas que tenían mucha escaldadura a los 15 días de esta fecha.

Se puede concluir que hay una diferencia significativa $(P<0.05)$ para el efecto de las hierbas. Con base en los totales, es claro que las semillas que se produjeron con hierbas, tienen la mitad de la germinación de las semillas que se produjeron en el lote limpio, y este puede ser un factor importante, pues afecta la producción, por otro lado, mientras menos semillas germinen, menos transmiten a $R$. secalis.

No hay una interacción de las hierbas por variedad, ya que el valor computado $\mathrm{F}$ (1.536 es menor que el valor tabulado de la $\mathrm{F}(1.99)(\mathrm{P}<0.05)$ (tabla 3). Este resultado significa que todas las variedades están afectadas en su germinación de semilla independientemente de los tratamientos (con y sin hierbas). En la tabla 4, se muestran los valores promedio de la germinación de la semilla de las variedades para cada uno de los 80 tratamientos. Hay diferencias significativas entre variedades en la capacidad de germinación de las semillas $(\mathrm{P}<0.05$. Las variedades que son estadísticamente significativas $(\mathrm{P}<0.05)$ porque tienen una mejor germinación son la línea 9626-B y las variedades Tlaxcala y Chevalier que también presentaron $R$. secalis (tabla 5).

TABLA 3. Análisis de varianza del efecto de los tratamientos con y sin hierbas sobre la germinación de semillas en invernadero.

Fuente de

variación
Grados de libertad
Suma de cuadrados
Cuadrados medios
Tratamientos

Variedad

Hierbas

Interacción

Variedad $\mathrm{x}$ hierbas

Error

60
19

9

1

9
6.54

4.64

1.02

0.88

3.82
0.3442

0.5156

0.098

n.S.*

1.0200

$16.021 \quad 0.05$

0.0978

1.536 n.s.*
F

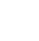


TABLA 4. Valores promedio de la germinación de semillas de las líneas / variedades para cada uno de los 80 tratamientos.

\begin{tabular}{|c|c|c|c|c|c|c|c|c|c|c|c|}
\hline \multirow[t]{2}{*}{ Variedad } & \multicolumn{4}{|c|}{$\begin{array}{l}\text { Libre de hierbas * } \\
\text { Repeticiones }\end{array}$} & \multirow[t]{2}{*}{$\begin{array}{l}\text { Presencia de } \\
\text { R. secalis }\end{array}$} & \multicolumn{4}{|c|}{$\begin{array}{l}\text { Con hierbas ** } \\
\text { Repeticiones }\end{array}$} & \multirow{2}{*}{$\begin{array}{l}\text { Presencia de } \\
\text { R. secalis }\end{array}$} & \multirow{2}{*}{$\begin{array}{l}\text { e Promedio } \\
\text { de las líneas } \\
\text { o variedades }\end{array}$} \\
\hline & 1 & 2 & 3 & 4 & & 1 & 2 & 3 & 4 & & \\
\hline 9554-II & 0.46 & 0.32 & 0.58 & 0.32 & + & 0.32 & 0.46 & 0.68 & 0.32 & + & 0.428 \\
\hline 9589-B & 0.46 & 0.32 & 0.32 & 0.00 & - & 0.32 & 0.00 & 0.00 & 0.00 & - & 0.178 \\
\hline 9591 & 0.00 & 0.32 & 0.00 & 0.46 & + & 0.00 & 0.00 & 0.00 & 0.00 & - & 0.098 \\
\hline 9592-G & 0.32 & 0.00 & 0.32 & 0.32 & - & 0.00 & 0.00 & 0.00 & 0.00 & - & 0.120 \\
\hline 9520-B & 0.58 & 0.46 & 0.68 & 0.00 & + & 0.00 & 0.46 & 0.32 & 0.89 & + & 0.424 \\
\hline 9626-B & 1.11 & 1.11 & 1.11 & 1.25 & + & 0.79 & 0.46 & 0.89 & 0.58 & + & 0.913 \\
\hline Porvenir & 0.46 & 0.89 & 0.46 & 0.46 & + & 0.32 & 0.00 & 0.00 & 0.32 & - & 0.364 \\
\hline Chevalier & 0.58 & 0.68 & 0.89 & 0.99 & + & 0.32 & 0.32 & 0.46 & 0.46 & + & 0.588 \\
\hline Tlaxcala & 0.46 & 0.46 & 0.46 & 0.89 & + & 0.32 & 0.00 & 1.25 & 1.25 & + & 0.636 \\
\hline $9414-4$ & 0.00 & 0.68 & 0.58 & 1.11 & + & 0.00 & 0.00 & 0.32 & 0.00 & - & 0.336 \\
\hline
\end{tabular}

* Con un total de $52.2 \%$ de promedio de semillas germinadas.

** Con un total de $29.5 \%$ de promedio de semillas germinadas. 
TABLA 5. Efecto de los tratamientos con y sin hierbas sobre la germinación de semillas.

Variedad o línea

de cebada
Media transformada**

para la germinación

en orden decreciente
Categorías de significancia*
9626-B

Tlaxcala

Chevalier

9554-I

9529-B

Porvenir

9514-A

9589-B

9592-G

9591
0.913

0.636

0.588

0.428

0.424

0.364

0.336

0.178

0.120

0.098 a

a b

a $\quad$ b $\quad c$

b $\quad$ c $\quad$ d

b c d

b $\quad$ c $\quad$ d

b c d

c d

d

d

* Las variedades con una letra en común no difieren estadísticamente $(P>0.05)$.

Las variedades que no tienen una letra en común son estadísticamente diferentes $(\mathrm{P}>0.05)$.

** Transformación angular de Bliss. 


\section{DISCUSIÓN}

En relación a los experimentos de deshierbe, es curioso observar que la línea 9591, a pesar de tener escaldadura en la semilla madre, casi no infectó ni a las hojas en el campo, ni a las semillas que se produjeron en los experimentos de invernadero; es posible que sea una línea resistente a la escaldadura. Las líneas 9626-B, 9592-G y la variedad Tlaxcala tenían semilla escaldada desde el inicio, así se mostró en hojas en el campo y en semillas en el invernadero. En estas 3 líneas y una variedad, el inóculo no sólo venía del rastrojo infectado, sino también en la semilla, mientras que el resto de las muestras sólo recibieron el inóculo del rastrojo; por eso no es de extrañar que la línea 9626-B y la variedad Tlaxcala fueran las más escaldadas en hojas y semillas, tanto en campo como en invernadero, y las que más transmitieron al hongo.

Como se puede apreciar en la tabla 1 y figura 1, hay una concordancia entre las líneas 9592-G y la 9626-B, que presentaron escaldadura en la semilla madre, con respecto a su comportamiento en el campo, donde fueron las únicas líneas con diferencia significativa $(\mathrm{P}<0.05)$ en el área foliar escaldada del lote limpio en relación con el enhierbado, y esta última línea (9626-B) fue la que presentó más escaldadura en invernadero. Pero no siempre hubo una concordancia tan clara, ya que la variedad Tlaxcala y la línea 9591, que tenían semilla madre escaldada, no se comportaron así. Aunque parece contradictorio este hecho, no lo es, pues no hay una relación entre la situación de que la semilla madre viniera o no escaldada, ya que hay que tomar en cuenta la resistencia o susceptibilidad de la línea o variedad a $R$. secalis, así lo demuestra la línea 9514-A, cuya semilla madre estaba sin escaldadura, y sin embargo tuvo mucha enfermedad en el campo y en el invernadero, que posiblemente provenía de esporas del aire o de rastrojo infectado del año previo.

Con respecto a la variedad Chevalier, en el campo tuvo poca escaldadura y en el invernadero hubo más, lo que se puede deber al efecto del hongo sobre la germinación de la semilla, que en esta variedad fue del $55 \%$, la más baja de todas. Hay que recordar que R. secalis baja la germinación de la semilla de la cebada.

Al analizar las condiciones climáticas de 1980, se ve que del 20 al 30 de enero hubo condiciones de alta precipitación y baja temperatura por 5 días, y ésto favoreció una buena infección causada por $R$. secalis, como se comprobó al medir el daño foliar en el campo, datos que apoyan los resultados de otros experimentos de epifitiología (Carvajal, 1986).

En el invernadero sí resulta evidente que las semillas provenientes del lote enhierbado tuvieron menos de la mitad de semillas con escaldadura que las del lote limpio, efecto que también se vió en el campo y que pudo deberse a dos hechos. El primero es que en el lote limpio no hay barreras mecánicas para que una espora salpique de una planta enferma a otra sana, mientras que las hierbas son una barrera para la transmisión. El segundo hecho es que las hierbas reducen el establecimiento de las plántulas de 
cebada, y al no haber plantas habrá menos transmisión por semilla, a veces la espiga no llega a formarse. En ambos casos la producción es baja.

Se puede decir que la transmisión de $R$, secalis por agua salpicada y por semilla fue más eficiente en las 40 parcelas limpias que en las enhierbadas, aunque hubo una mejor germinación de la semilla.

AGRADECIMIENTOS. A Jesús Rojo y Juan López por ayudar con el arado de las tierras, la siembra y la cosecha de la cebada. Al Campo Agrícola Experimental del Valle de México, "El Horno" del Instituto Nacional de Investigaciones Agrícolas (INIA) por facilitar las parcelas. Al Centro Internacional de Mejoramiento de Maíz y Trigo (CIMMYT) por facilitar datos sobre las condiciones climáticas del experimento.

\section{LITERATURA CITADA}

CARVAJAL, M. 1986. Estudio sobre la biología, epifitiología y control de Rhynchosporium secalis (Oud.) Davis, hongo causante de la escaldadura de la cebada. Tesis de Doctorado. Facultad de Ciencias, Universidad Nacional Autónoma de México. 204p.

CARVAJAL, M., E. RiOJAS GuAdiAnA, y M. MENDOZA, 1986. Efecto de la densidad de siembra sobre la transmisión de la escaldadura de la cebada en México. Rev. Mex. Mic. 2:239-250.

HABGOOD, R.M. 1971. The transmission of Rhynchosporium secalis by infected barley seed. Pl. Path. 20:80-81.

KAY, J.G. 1971. Physiologic especialization and seed transmission of Rhynchosporium secalis. PhD. Thesis. University of Reading.

MinisTRY OF AGRICULTURE, FiSHERIES AND FOOD. 1972. Leaf blotch and halo spot of barley. Advisory leaflet. United Kingdom, 580:2-5

OzOE, S. 1956. Studies on the Rhynchosporium scald of barley and its control. Bull. Shimane. Agric. Coll. 1:1-122.

REED, H.E. 1957. Studies on barley scald. Bull. Tennessee Univ. Agric. Exp. Stn. No. 268:42.

SKOROPAD, W.P. 1959. Seed and seedling infection of barley by Rhynchosporium secalis. Phytopathology 49:623-626.

SKOROPAD, W.P. 1960. Barley scald in the prairies provinces of Canada. Com. Phytopath. News. 6:25-27. SMITH, N.J.H. 1937. Leaf scald of barley in South Africa. S. Afr. J. Sci. 34:286-290.

ZAR, J.H. 1974. Biostatistical analysis. Prentice-Hall, Inc., Englewood Cliffs, N.J. 620p. 